\title{
Simulation of 3-dimensional Morphology on Different Substrates of Thin Film Growth
}

\author{
Wu Lili \\ XingZhi college of Zhejiang Normal University, \\ goiuy@zjnu.cn
}

\begin{abstract}
A kinetic Monte Carlo model describing the three-dimensional thin film growth on different substrate $s$ is presented in which the diffusion of adatoms on substrate and between various layers as well as the exchange process between diffusing adatioms and the underneath surfactant atoms are taken into account. The influences of the ES barrier, the shape of substrate and the surfactant-covered substrate on the growth mode and morphology during the multi-layer film growth are studied. The relationship between surface roughness and the coverage are considered in different substrate conditions.
\end{abstract}

Keywords-film growth, substrate, exchange, monte carlo simulation, roughness

\section{INTRODUCTION}

Thera are many experiments and theories are developed to get layer-by-layer (LBL) smooth surface in thin film both in metal or semiconductor surface, such as on the heterogeneous surface[1-4], the surfactant-preoccupied substrate[5-12] and so on. To understand the detailed atomic process during the thin growth, it is worthwhile to study using a Monte Carlo simulation by computer. Simualtion of film growth on different surface is important since the substrate conditions will influence the quality of the thin film. Here we formulate such a model in which the effects of the ES barrier, the shape of substrate and the surfactant-covered substrate on the growth mode and 3-dimensional morphologies during the multi-layer film growth are considered.

\section{Simulation MODEL}

The simulation were carried out on a $100 \times 100$ substrate. Adatoms were depostied on the substrate with the flux rate $F=0.02 M L / s$ (monolayer/s). The arrived adaomts were migrating on the substrate. Surface migration is modeled as a nearest-neighbor hopping process, with the hopping rate of an Arrehnius form:

$$
D=D_{0} \exp \left(-E / k_{B} T\right)
$$

Where $D_{0}$ is the vibration frequency of a surface adatom and we assume it is a constant $\left(=10^{13} s^{-1}\right) ; k_{B}$ is Boltzmann's constant, $T$ is the surface temperature, $T=300 K$ is used in our model and $E$ is an energy barrier to hop. The energy barrier in our model are

\author{
Zhang Junying \\ Shanghai Technical Institute of Electronics \& Information \\ junying_zh@163.com
}

consist of two parts: the $E_{d}=0.08 \mathrm{eV}$ barrier for in-layer diffusion and the ES barrier for inter-layer transportion, i.e.,

$$
E=E_{d}+\left(m_{i}-m_{f}\right) \Theta\left(m_{i}-m_{f}\right) E S
$$

Where $m_{i}$ and $m_{f}$ are the number of next-nearest neighbors in the plane beneath before $\left({ }^{m_{i}}\right)$ and after( $\left.{ }^{m_{f}}\right)$ the hop. The Heaviside function $\Theta(x)=1$ if $x>0$, and 0 otherwise.The ES barrier has a nonzero value only if $m_{i}>m_{f}$ and equals $\left(m_{i}-m_{f}\right) E S_{\text {.[13,14], There are }}$ four nearest neighbors on the squere lattice substrate and six on the triangular lattice substrate.

When the substrate are pre-covered with certain covrage of surfactant atoms in some metal or semiconductor surface. The exchange process will happen when a diffusing adatom is just on top of a surfactant atom and can overcome the exchange barrier $E_{e x}$, it will be dropped to the beneath site, on the other hand, the surfactant atom will be lifted to the above site,with the rate of an Arrehnius form[15]:

$$
D_{e x}=D_{0} \exp \left(-E_{e x} / k_{B} T\right)
$$

In order to accurately determine the growth mode during multi-layer thin film growth, the surface roughness as a function of coverage is calculated. The surface roughness is defined as :[1,17]

$$
\omega=\sqrt{\frac{1}{n} \sum_{i=1}^{n}\left(h\left(x_{i}\right)-\bar{h}\right)^{2}}
$$

Where $n$ is the total number of deposited atoms, $h\left(x_{i}\right)$ is the height of thin film at the position of ${ }^{x_{i}}$ and $\bar{h}$ is the average height of thin films, which is giver by $\bar{h}=\left(\frac{1}{n}\right) \sum_{i=1}^{n} h\left(x_{i}\right)$

In our model desorption is neglected and the critical island size is set to 1 , which means the dimmer is stable and can absorb the monomers to form a new island. The periodic boundary condition is considered.

\section{RESUlts AND Dicussion}

Figure.1.(a)-(d) show the 3D morphologies of adatoms deposited on the trianguler subtrate without surfactant pre-occupied. The growth mode and the morphologies in multi-layer growth of thin film dependent on the ES barrier 
which determine the inter-layer transiportion. As we have seen in Figure.1(a)-(d), when the ES barrier decreased from $0.15 \mathrm{eV}$ to $0.01 \mathrm{eV}$, the barrier for adatoms to overcome to drop decreased. As a result, inter-layer transiport enhanced and the grwoth mode is transited from Volmer-Weber( 3D) to Frank-van der Merwe(Layer-By-Layer) after $4 \mathrm{ML}$ atoms deposited. The coverage dependence of surface roughness of Figure.1(a)-(d) is showed in Figure.2. The roughness of Figure.1(a)-(b) grows with the coveragae indicating a 3D growth mode. Obviously, when the roughness oscillates with the coverage in Figure.2, it shows the LBL growth mode in Figure.1(c)-(d).

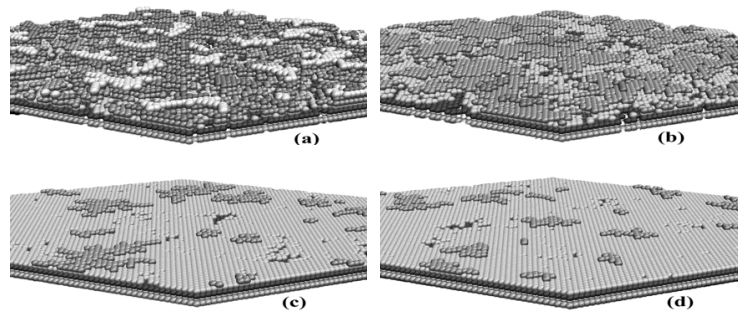

Figure 1. Surface morphologies obtained after growing 4 ML of A atoms in triangular substratee with no surfactant-covered under different $\mathrm{ES}$ barrier (a) $\mathrm{ES}=0.15 \mathrm{eV}$, (b) $\mathrm{ES}=0.1 \mathrm{eV}$, (c) $\mathrm{ES}=0.05 \mathrm{eV},(\mathrm{d}) \mathrm{ES}=0.01 \mathrm{eV}$ under other identical growth conditions. The spheres of different colors in (a)-(d) represent adatoms in different layers.

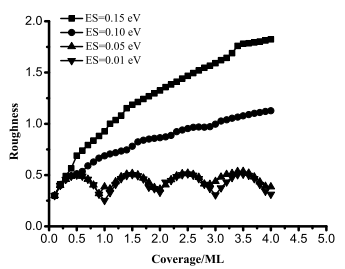

Figure 2. Coverage dependence of surface roughness at different ES barriers, i.e.ES $=0.15,0.1,0.05$, and $0.01 \mathrm{eV}$. (a)

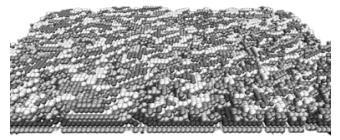

(c)

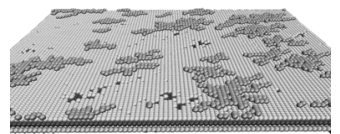

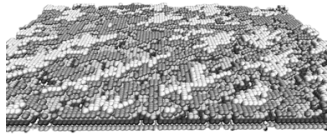

(d)

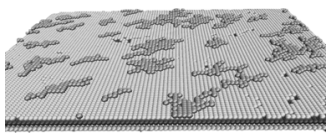

Figure 3. Surface morphologies obtained after growing 4 ML of adatoms in square substrate with no surfactant-covered under different ES barrier (a)ES $=0.15 \mathrm{eV}$, (b) $\mathrm{ES}=0.1 \mathrm{eV},(\mathrm{c}) \mathrm{ES}=0.05 \mathrm{eV},(\mathrm{d}) \mathrm{ES}=0.01 \mathrm{eV}$ under other identical growth conditions. The spheres of different colors in (a)-(d) represent adatoms in different layers.

The triangular lattice is hexagon substrate and each adatom has six nearest neighbors. Most of the fcc(111)substrates such as $\operatorname{Ag}(111), \operatorname{Pt}(111)$ and $\operatorname{Si}(111)$, are triangular. There are also many square shape substrates in the film system, for example the $\mathrm{Si}(001)$ and $\mathrm{Cu}(001)$ substrate. Figure.3. shows the 3D morphologies of thin film growth on the square substrate with different ES barrier. As we can see, the surface is rough when the ES are bigger in Figure.3.(a)-(b) and is smooth as the ES decreases in Figure3.(c)-(d). The dependence of roughness on coverage of Figure.3. are similar with the Figure.2. That is say the effects of ES barrier on the growth mode of square substrate are the same with the growth on triangular substrate. (a)

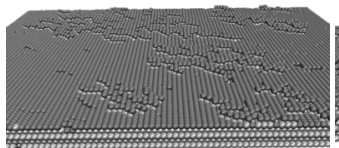

(c)

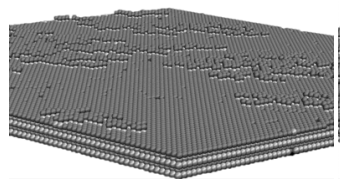

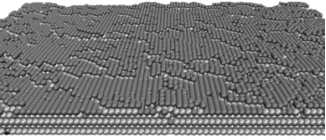

(d)

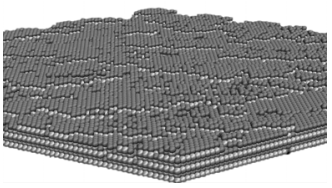

Figure 4. The morphologies of thin film growth on monolayer surfactant covered of square and triangular semiconductor substrates with different exchange barriers: (a)and(c) $E_{e x}=0.08 \mathrm{eV}$;(b)and(d)

$E_{e x}=0.12 \mathrm{eV}$.The darker spheres float on the top are the surfactant atoms; the gray spheres present the adatoms of different layers.

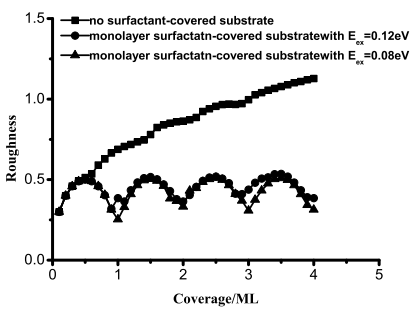

Figure 5. The surface roughness' evolutions with the coverage obtained in simulations on no surfactant substrate and mono-layer surfactant-covered substrate with decreased exchange barrier, i.e. $E_{e x}=0.12 \mathrm{eV}, 0.08 \mathrm{eV}$.

Surfactants can modify the growth of thin film epitaxial layers on metal or semiconductor substrates. With surfactants, a layer-by-layer(LBL) growth can be achieved, this is of great importance for fabricating advanced electronic devices. Usually, a monolayer of surfactant is initially deposited onto the semiconductor substrate and a submonolayer of surfactant is pre-deposited onto the metal substrate. It has been shown that different surfactants may modify the epitaxial growth in quite a different way, but the exchange process will always happen when a diffusing adatom is just on top of a surfactant atom and can overcome the exchange barrier $E_{e x}$, it will be dropped to the beneath site, on the other hand, the surfactant atom will be lifted to the above site. When the semiconductor substrate of square 
shape covered with a monolayer surfactants, the morphologies of 4ML growth is show in Figure.4.(a)-(b) with different exchange barrier. The ES barrier used in our model is $\mathrm{ES}=0.15 \mathrm{eV}$ when we discuss the effect of surfactant in multi-layer thin film growth.

When the surfactant was deposited on the metal surface to modify the growth mode of thin film epitaxial growth, submonolayer of surfactant is needed. As we have discussed, the exchange process between deposited adatoms and the pre-occupied surfactant atoms are considered. The exchange mechanism will enhanced the inter-layer transport thus the growth mode trend to LBL growth if the exchange barrier is smaller than the ES barrier on the model. Figure.6.demonstrate the 3D morphologies which were obtained in our model when the square and triangular substrates with $\theta_{M}=0.03 M L$ and $\theta_{M}=0.08 M L$ surfactant atoms pre-covered under the same other conditions $E_{e x}=0.08 e V$ and $\mathrm{ES}=0.15 \mathrm{eV}$.
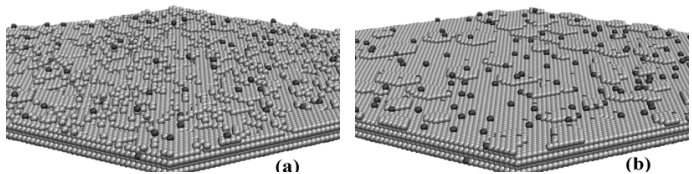
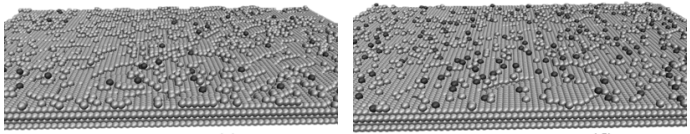

(d)

Figure 6. The morphologies of thin film growth on submono-laye surfactant covered of square and triangular metal substrates with different surfactant coverage: (a)and(c) $\theta_{M}=0.03 M L$;(b)and(d) $\theta_{M}=0.08 M L$. The

darker spheres are the surfactant atoms; the gray spheres present the adatoms of different layer.

The film surface in Figure.6.(b) is smoother than that in Figure.6(a) when the coverage of surfactant increase from $0.03 \mathrm{ML}$ to $0.08 \mathrm{ML}$, that is to say, with the same exchange barrier, the substrates with different coverage will affect the quality of thin film. The influence of the surfactant coverage on the growth mode has been discussed our other papers[18]

\section{SUMMARIES}

A kinetic Monte Carlo simulation model has been presented to investigate the 3D morphology of multi-layer thin film growth on different conditions' substrates. The influences of the ES barrier, the shape of substrate and the surfactant-covered substrate on the growth mode and morphology during the multi-layer film growth are studied. Our finds are helpful to develop nano-technology in film growth.

\section{REFERENCES}

[1] H.J.Lu,F.M.Wu and Y.Z.Fang, Chin Phys.13,2004,PP:2038-2044.

[2] E.G. Wang, Progress in Phys. 23 ,2003,PP:22-27.

[3] T.L.Hill.,J Chem Phys.17,1949,PP:520-535.

[4] Nichcholas Metropolis,Arianna W.Rosenbluth,Marshall N.Rosenbluth and Edware eller, J Chem Phys.21,1953,PP:1.87-1092.

[5] J. Vrijmeoth, H.A. van der Vegt, J.A. Meyer, E. Vlieg, and R.J. Behm, Phys. Rev. Lett. 72 ,1994,PP:3843-3848.

[6] H. Wolter, M. Schmidt, and K. Wandelt, Surf. Sci. 298 ,1993,PP:173-177.

[7] J. Wu, B.G. Liu, Z.Y. Zhang, and E.G. Wang, Phys. Rev. B 61 , 2000,PP:13212-13217..

[8] Matthias Kal, George Comsa, and George Comsa, Phys. Rev. Lett. 81 (1998) 1255

[9] I. Markov, Phys. Rev. B 50 (1994) 11271

[10] D. Kandel, Phys. Rev. Lett. 78 ,1997,PP:499-506.

[11] Z. Zhang and M.G. Lagally, Phys. Rev. Lett. 72 , 1994,PP:693-698.

[12] B.G. Liu, J. Wu, and E.G. Wang, Phys. Rev. Lett. 83 , 1999,PP:1195-1199.

[13] P. Smilaner and D.D. Vvedensky, Phys. Rev. B 52 , 1995,PP:14263-14268.

[14] J. KallumKi and J. Krug, Phys. Rev. B 65 ,2002,PP:205411-205416.

[15] Dai-Mu Wang, Ze-Jun Ding, and Xia Sun, Phys. Rev. B 72 ,2005,PP:115419-115426.

[16] F.M. Wu, H.J. Lu, and Z.Q. Wu, Chin. Phys. 15 ,2006,PP:807-812.

[17] L L Wu and F M Wu, Chin.Phys.B,19 ,2010,PP:066801-066807. 